\title{
Anti-B7H3 Antibody-drug Conjugate MGC018
}

National Cancer Institute

\section{Source}

National Cancer Institute. Anti-B7H3 Antibody-drug Conjugate MGC018. NCI Thesaurus. Code C159495.

An antibody-drug conjug ate (ADC) comprised of an anti-B7-homolog 3 (B7-H3, CD276) humanized immunoglobulin G1 (IgG1)/kappa monoclonal antibody conjug ated through reduced interchain disulfides to the cleavable linker-duocarmycin payload, valine-citrullineseco DUocarmycin hydroxyBenzamide Azaindole (vc-seco-DUBA), with potential antineoplastic activity. Upon administration, the antibody moiety of anti-B7-H3 ADC MGC018 specifically targets and binds to the cell surface antigen B7-H3, leading to internalization of the ADC by the tumor cell. The linker is cleaved inside the tumor cell by proteases at the dipeptide valine-citrulline (vc), thereby releasing the duocarmycin payload. Duocarmycin binds to the minor groove of DNA, alkylates adenine at the N3 position, and induces cell death. $\mathrm{B} 7-\mathrm{H} 3$, a type I transmembrane protein and a member of the B7 co-stimulatory protein superfamily, is overexpressed on certain tumor cell types and on various immune cells but is minimally expressed by normal human tissues. B7-H3 is a negative regulator of T-cell activation and its overexpression plays a key role in immuno-evasion, tumor cell invasion and metastasis, and its expression is correlated with poor prognosis. 